\title{
SB1518, a novel macrocyclic pyrimidine-based JAK2 inhibitor for the treatment of myeloid and lymphoid malignancies
}

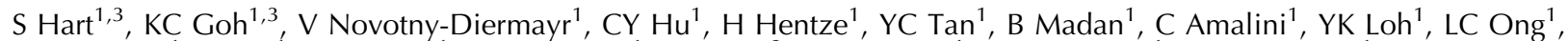

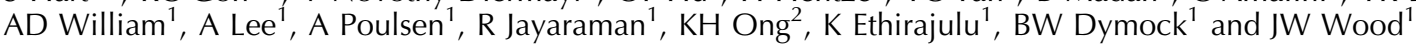

${ }^{1} S^{*}$ BIO Pte Ltd, Singapore, Singapore and ${ }^{2}$ Tan Tock Seng Hospital, 11 Jalan Tan Tock Seng, Singapore, Singapore

\begin{abstract}
SB1518 is an innovative pyrimidine-based macrocycle that shows a unique kinase profile with selective inhibition of Janus Kinase-2 (JAK2; $\mathrm{IC}_{50}=23$ and $19 \mathrm{nM}$ for JAK2 ${ }^{\mathrm{WT}}$ and JAK2 ${ }^{\mathrm{V} 617 \mathrm{~F}}$ respectively) within the JAK family $\left(\mathrm{IC}_{50}=1280,520\right.$ and $50 \mathrm{nM}$ for JAK1, JK3 and TYK2, respectively) and fms-like tyrosine kinase-3 $\left(F L T 3 ; I_{50}=22 \mathrm{nM}\right)$. SB1518 shows potent effects on cellular JAK/STAT pathways, inhibiting tyrosine phosphorylation on JAK2 (Y221) and downstream STATs. As a consequence SB1518 has potent anti-proliferative effects on myeloid and lymphoid cell lines driven by mutant or wild-type JAK2 or FLT3, resulting from cell cycle arrest and induction of apoptosis. SB1518 has favorable pharmacokinetic properties after oral dosing in mice, is well tolerated and significantly reduces splenomegaly and hepatomegaly in a JAK2 ${ }^{\mathrm{V} 617 \mathrm{~F}}$-driven disease model. SB1518 dose-dependently inhibits intra-tumor JAK2/ STAT5 signaling, leading to tumor growth inhibition in a subcutaneous model generated with SET-2 cells derived from a JAK2 $^{\mathrm{V} 617 \mathrm{~F}}$ patient with megakaryoblastic leukemia. Moreover, SB1518 is active against primary erythroid progenitor cells sampled from patients with myeloproliferative disease. In summary, SB1518 has a unique profile and is efficacious and well tolerated in JAK2-dependent models. These favorable properties are now being confirmed in clinical studies in patients with myelofibrosis and lymphoma.

Leukemia (2011) 25, 1751-1759; doi:10.1038/leu.2011.148;
\end{abstract}

published online 21 June 2011

Keywords: SB1518; JAK2; myelofibrosis; lymphoma

kinase inhibitor

\section{Introduction}

The Janus Kinase (JAK) family consists of four cytoplasmic protein tyrosine kinases that have important roles in the cellular signaling pathways that control proliferation, differentiation and cell death. ${ }^{1}$ Genetic lesions and aberrations involving JAK2 have been found to be associated with a wide spectrum of hematological malignancies, including myelofibrosis, lymphomas and some rare leukemias. ${ }^{2}$ FLT3 (fms-like tyrosine kinase-3) belongs to a family of class III receptor tyrosine kinases and it is the most frequently mutated gene in acute myeloid leukemia leading to poor prognosis in some patients. ${ }^{3}$ As myelofibrosis and acute myeloid leukemia continue to present as unmet medical needs in hematology, JAK2 and FLT3 offer hope as novel targets for the development of innovative therapies.

Macrocyclic natural products have evolved to interact with proteins, by virtue of their innate potential for diverse

Correspondence: Dr S Hart, S*BIO Pte Ltd, 1 Science Park Road, \#05-09 The Capricorn, Singapore 117528, Singapore.

E-mail: stefan_hart@sbio.com

${ }^{3}$ These authors contributed equally to this work.

Received 11 February 2011; revised 21 April 2011; accepted 9 May 2011; published online 21 June 2011 functionalization and stereochemical disposition within a conformationally pre-defined ring structure. ${ }^{4}$ As such, macrocyclic organic compounds in general constitute a structural class that possesses immense potential for pharmacological applications, but their utility has not been fully exploited because of the synthetic challenges and concerns over apparent lack of 'druglikeness'. Recent advances in synthetic medicinal chemistry are beginning to change this scenario. ${ }^{4}$

Herein, we report for the first time the structure and the pharmacological profile of SB1518, a novel low-molecularweight macrocycle with potent inhibitory activities against JAK2 and FLT3. SB1518 exhibits favorable pharmaceutical properties and shows efficacy in cellular and animal models of hematological malignancies as well as primary cells derived from patients with myeloproliferative disease. SB1518 has shown promising clinical activity in phase $1 / 2$ trials in advanced myeloid and lymphoid malignancies and is currently under evaluation in multi-center phase 2 clinical trials for myelofibrosis and lymphomas. ${ }^{5,6}$

\section{Materials and methods}

Compounds and reagents

SB1518, as depicted in Table 1: 11-(2-pyrrolidin-1-yl-ethoxy)14,19-dioxa-5,7,26-triaza-tetracyclo(19.3.1.1(2,6).1(8,12))heptacosa-1(25),2(26),3,5,8,10,12(27),16,21,23-decaene, was discovered and synthesized by $\mathrm{S} * \mathrm{BIO}$ Pte Ltd (Singapore, Singapore). INCB018424 and TG101348 were synthesized at Sai Advantium Pharma Ltd (Hyderabad, India). JAK inhibitor-I, a pan-JAK inhibitor, was obtained from Calbiochem (San Diego, CA, USA; Cat. No. 420099). For in vivo studies, dosing solutions were prepared in $0.5 \%$ methylcellulose $(w / v)$ and $0.1 \%$ Tween80 in $\mathrm{H}_{2} \mathrm{O}$ (MC/Tween). Interferon- $\alpha$, interferon- $\gamma$, interleukin-2 and interleukin-3 were purchased from i-DNA (Singapore, Singapore).

In vitro kinase assays

For details, see Supplementary information.

\section{Cell culture and proliferation assay}

SET-2 and Karpas 1106P cells were obtained from DSMZ (Braunschweig, Germany). Ba/F3-JAK2 ${ }^{\mathrm{V} 617 \mathrm{~F}}$-GFP-Luc cells were a gift from Dr Martin Sattler (Dana-Farber Cancer Institute, Boston, MA, USA) and have been described previously. ${ }^{7}$ All other cells were obtained from the American Type Culture Collection (ATCC, Manassas, VA, USA) and cultured according to the recommended guidelines. For proliferation assays in 96-well plates, cells were seeded at $30-50 \%$ confluency and were treated the following day with compounds (in triplicate) at 


Selectivity
vs JAK2

11-(2-Pyrrolidin-1-yl-ethoxy)-14,19-dioxa-5,7,26-triazatetracyclo[19.3.1.1(2,6).1(8,12)]heptacosa1(25),2(26),3,5,8,10,12(27),16,21,23-decaene

Abbreviations: JAK, Janus Kinase.

Values (mean \pm s.d.) were obtained from $\mathrm{S}^{\star} \mathrm{BIO}$ in-house assays $(n>3)$ except the mutant kinases tested at *Millipore $(n=1)$.

concentrations up to $10 \mu \mathrm{M}$ for $48 \mathrm{~h}$. Cell viability was monitored using the CellTiter-Glo assay (Promega, Madison, WI, USA). Dose-response curves were plotted to determine $\mathrm{IC}_{50}$ values for the compounds using the XL-fit software (IDBS Ltd, Alameda, CA, USA).

\section{Collection and processing of human blood samples for peripheral blood mononuclear cell (PBMC) purification and erythroid progenitor expansion} Blood collection was approved by the Institutional Review Board, Tan Tock Seng Hospital, Singapore (DSRB-8/08/15). All subjects (patients with polycythemia vera (PV) or normal volunteers) provided informed consent. Blood $(15 \mathrm{ml})$ was drawn by venipuncture into 8-ml BD vacutainer CPT (BD Bioscience, Franklin Lakes, NJ, USA). PBMCs were isolated according to the manufacturer's instructions and stored at $-80^{\circ} \mathrm{C}$. Expansion of erythroid progenitor cells (EPCs) from PBMCs sampled from patients with PV or normal volunteers was performed according to a published 2-step protocol by Manshouri et al. ${ }^{8}$ On day 12 , cells were treated for $48 \mathrm{~h}$ with compounds and cell viability was measured with CellTiter-Glo assay.

Colony-forming assay (endogenous erythroid colony) A total of $2-8 \times 10^{5}$ PBMCs from PV patients were plated onto minimal H4531 Methocult medium (StemCells Technology, Vancouver, Canada) with indicated concentrations of SB1518 or DMSO. Each condition was performed in triplicate. After 14 days, colonies derived from erythroid (BFU-E and CFU-E) and myeloid (CFU-M and CFU-GM) progenitor cells were counted.

\section{Western blot analysis}

Cells were lysed and proteins immunoprecipitated as described previously. ${ }^{9}$ Following SDS-polyacrylamide gel electrophoresis, proteins were transferred to polyvinylidene fluoride membranes. Western blots were performed according to standard methods. pJAK2 (Y1007/8; Cat. No. 3776), pJAK2 (Y221; Cat. No. 3774), pJAK1 (Y1022/3; Cat. No. 3331), pSTAT1 (Y701; Cat.
No. 9167), pSTAT3 (Y705), phospho-tyrosine (pY; No. 9411), anti-mouse immunoglobulin G (Cat. No. 7076) and anti-rabbit immunoglobulin G, HRP-linked (Cat. No. 7074) antibodies were purchased from Cell Signaling Technology (Beverly, MA, USA). pSTAT5 (Y694, Cat. No. 611965) was obtained from BD Biosciences (San Jose, CA, USA), tubulin (Cat. No. 65-829) from Millipore (Billerica, MA, USA), JAK2 (Cat. No. SC-278) from Santa Cruz Biotechnology (Santa Cruz, CA, USA) and actin (Cat. No. A2066) from Sigma (St Louis, MO, USA). The images were captured digitally using the LAS-3000 Life Science Imager from Fujifilm (Tokyo, Japan). Densitometric analysis was performed using the MultiGauge software (v3.1) from Fujifilm.

\section{Flow cytometry}

For cell cycle analysis, $5 \times 10^{5} / \mathrm{ml} \mathrm{SET-2}$ or Karpas $1106 \mathrm{P}$ cells were treated for $24 \mathrm{~h}$ with SB1518 at $1 \times$ and $3 \times$ the $\mathrm{IC}_{50}$ for cell proliferation. After treatment, cells were fixed using $70 \%$ ice-cold ethanol and stained with propidium iodide.

For the analysis of expanded erythroid progenitors, FITCconjugated anti-human-CD71 (transferrin receptor, Cat. No. 555536) and allophycocyanin conjugated anti-CD235A (glycophorin-A, Cat. No. 551336, BD Biosciences) monoclonal antibodies were used for staining the cells at day 12. All stained cells were analyzed on the FACSCalibur equipped with the CellQuest Pro software (BD Systems, San Jose, CA, USA).

\section{In vivo efficacy studies}

Female athymic BALB/c nude mice (BALB/cOlaHsd-Foxn $1^{n u}$ ) of age 12 weeks were obtained from the Biological Research Centre (Biopolis, Singapore, Singapore); female SCID Beige mice (CB17.Cg-Prkd $C^{\text {scid } L y s t ~}{ }^{\text {bg/Crl) }}$ of age 9-10 weeks were obtained from BioLASCO (Taipei, Taiwan). Standard protocols were followed, in compliance with the NIH and the National Advisory Committee for Laboratory Animal Research guidelines (IACUC approval No. 0800371).

For the mouse model of myeloproliferative neoplasm, $2 \times 10^{6}$ $\mathrm{Ba} / \mathrm{F} 3-\mathrm{JAK} 2^{\mathrm{V} 617 \mathrm{~F}}$ cells were resuspended in $100 \mu \mathrm{l}$ serum-free culture medium and injected intravenously into the tail vein of 
BALB/c nude mice. Treatment was started on day 4 for 14 consecutive days for three groups of 10 mice each. Four naive mice served to establish baseline values. Blood counts were determined with the ScilVET abc hematology analyzer (Scil Animal Care Company, Viernheim, Germany).

For the SET-2 leukemia model, $5 \times 10^{6}$ tumor cells were injected subcutaneously in the right flank of severe combined immunodeficient beige mice. The cells were resuspended in $50 \mu \mathrm{l}$ serum-free growth medium, mixed 1:1 with Matrigel (Cat. No. 354248, BD Biosciences) and injected in a total volume of $100 \mu \mathrm{l}$. Tumor volumes were determined by caliper measurements and drug treatment was initiated after 31 days when tumors had reached a mean volume of $280 \mathrm{~mm}^{3}$ (tumor volume $\left(\mathrm{mm}^{3}\right)=\left(w^{2} \times I\right) / 2(w$, width and $I$, length of the tumor in $\left.\mathrm{mm})\right)$. This study was performed using 12 mice per group and animals were killed $3 \mathrm{~h}$ postdose on day 18 . Tumor growth inhibition was calculated as described previously. ${ }^{10}$ For the efficacy studies, mice were treated by oral gavage $(10 \mathrm{ml} / \mathrm{kg}$ body weight) with doses from 50 to $150 \mathrm{mg} / \mathrm{kg}$ SB1518.

\section{Statistics}

Statistical analysis was performed using GraphPad Prism v5 Software. The statistical test applied for particular experiments is shown in each figure legend.

\section{Results}

\section{Kinase selectivity spectrum of SB1518}

SB1518 was designed with the aid of computational chemistry as a small-molecule adenosine triphosphate-site competitive inhibitor of JAK2 kinase with favorable physico-chemical and pharmaceutical properties. It contains a pyrimidine core within a low-molecular-weight macrocyclic structure (Table 1). It was subsequently tested against $>50$ other protein kinases covering all major families of the human protein kinome.

SB1518 is a potent inhibitor of both wild-type JAK2 $\left(\mathrm{IC}_{50}=23 \mathrm{nM}\right)$ and $\mathrm{JAK} 2^{\mathrm{V} 617 \mathrm{~F}}$ mutant $\left(\mathrm{IC}_{50}=19 \mathrm{nM}\right)$ that is present in high frequencies among patients with MPD (Table 1). Relative to JAK2, SB1518 is two-fold less potent against TYK2 $\left(\mathrm{IC}_{50}=50 \mathrm{nM}\right), 23$-fold less potent against JAK3 $\left(\mathrm{IC}_{50}=520 \mathrm{nM}\right)$ and 56-fold less potent against JAK1 $\left(\mathrm{IC}_{50}=1280 \mathrm{nM}\right)$. SB1518 also inhibits FLT3 $\left(\mathrm{IC}_{50}=22 \mathrm{nM}\right)$ and its mutant FLT3-D835Y $\left(\mathrm{IC}_{50}=6 \mathrm{nM}\right)$. The rest of the evaluated kinases showed $<30 \%$ inhibition when tested against $100 \mathrm{nM}$ SB1518 at adenosine triphosphate concentrations equivalent to its Michaelis constant ( $K_{\mathrm{m}} ;$ Supplementary information).

\section{Modulation of JAK2 signaling pathways by SB1518}

To investigate whether the enzyme-inhibitory properties of SB1518 translate into modulation of JAK2 signaling pathways, the phosphorylation status of JAK2 and downstream substrates was determined in several JAK2 ${ }^{\mathrm{WT}}$ - and JAK2 ${ }^{\mathrm{V} 617 \mathrm{~F}}$-harboring cells.

First, the effects of SB1518 on ligand-dependent JAK2 signaling in human cell lines endogenously expressing the wild-type JAK2 were examined. Cognate binding of the interferon- $\gamma$ receptors in these cells causes autophosphorylation of JAK2 on Y1007/8 and activation of different STAT proteins depending on the cell types. ${ }^{11}$ SB1518 induced concentrationdependent inhibition of phosphorylation on JAK2, STAT3 and STAT5 in 293T (human embryonic kidney cells; Figure 1a), whereas a parallel effect on STAT1 was shown in Jurkat cells (Figure $1 \mathrm{~b})$, which are of lymphoid origin.

Next, the effect of SB1518 on ligand-independent JAK2 ${ }^{\text {WT }}$ signaling was studied in the human mediastinal lymphoma cell line, Karpas 1106P. In this cell line, STAT3 is present in a state of high basal phosphorylation because of constitutive JAK2 signaling caused by the genetic deletion of its negative regulator suppressor of cytokine signaling (SOCS)-1. ${ }^{12}$ Exposure of Karpas $1106 \mathrm{P}$ cells to SB1518 for $3 \mathrm{~h}$ led to attenuation of the basal level of pSTAT3 (Y705; Figure 1c).

To determine whether the in vitro JAK2/JAK1 selectivity of SB1518 translates into differential effects in the cellular context, we investigated the autophosphorylation on these 2 kinases in Jurkat cells, which had been treated with the broad-spectrum a

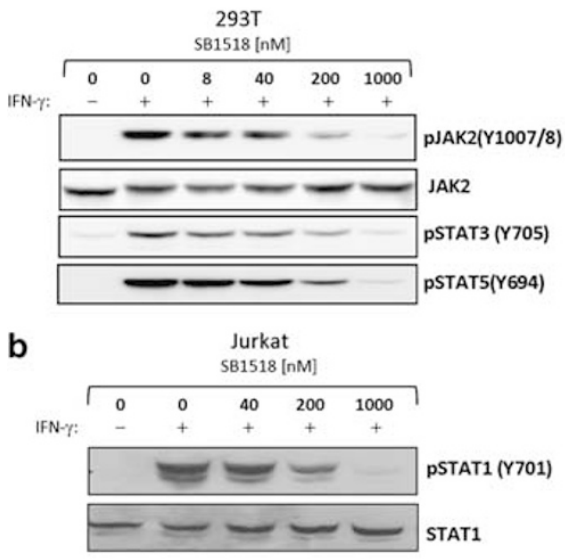

C
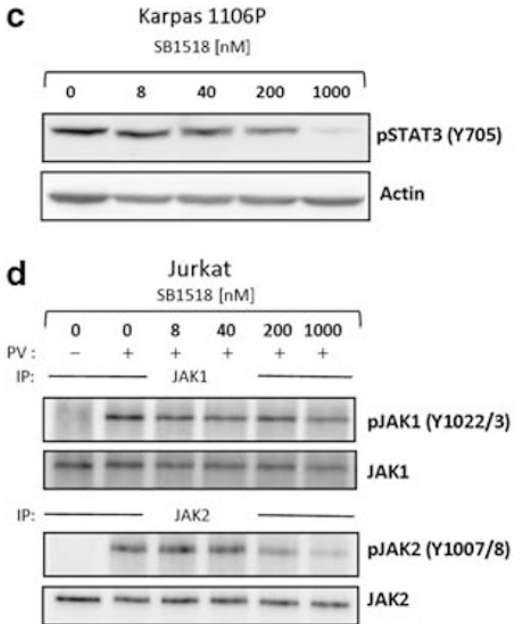

Figure 1 SB1518 effectively blocks signaling pathways in JAK2 ${ }^{\mathrm{WT}}$-expressing cell lines. (a) 293T (b) Jurkat and (c) Karpas $1106 \mathrm{P}$ cells were pretreated with SB1518 for $3 \mathrm{~h}$ and 293T, and Jurkat cells were treated for $10 \mathrm{~min}$ with $100 \mathrm{ng} / \mathrm{ml}$ interferon- $\gamma$ as indicated. After lysis, pJAK2 (Y1007/8) was detected by immunoblotting (IB) with anti-p(1007/8)JAK2 antibody. pSTAT1, pSTAT3 and pSTAT5 were detected by IB with antip(Y701)STAT1, anti-p(Y705)STAT3 or anti-p(694)STAT5 antibody, respectively. As a loading control, the same membranes were re-probed with anti-JAK2, anti-STAT1 or anti-actin antibody. (d) Jurkat cells were pre-treated with SB1518 for $3 \mathrm{~h}$ and were treated with $5 \mathrm{ng} / \mathrm{ml}$ pervanadate for $10 \mathrm{~min}$ as indicated. After lysis, JAK1 and JAK2 were immunoprecipitated and their phosphorylation status was determined by immunoblotting using phospho-specific antibodies. The same membranes were re-probed with anti-JAK1/or JAK2 antibody to detect the total protein levels. 
phosphatase inhibitor pervanadate. Consistent with potencies from the in vitro kinase screen, pJAK2 was significantly inhibited at lower concentrations than pJAK1 (Figure 1d).

In addition to the effects of SB1518 on JAK2 ${ }^{\mathrm{WT}}$ signaling, studies were performed on two cell lines expressing endogenous JAK2 ${ }^{\mathrm{V} 617 \mathrm{~F}}$ (HEL92.1.7, a human erythroleukemia cell line homozygous for JAK2 ${ }^{\mathrm{V} 617 \mathrm{~F}}$ and SET-2, a human essential thrombocythemia cell line heterozygous for JAK2 ${ }^{\mathrm{V} 617 \mathrm{~F}}$ ) and a murine pro- $B$ cell line transformed by exogenously expressed JAK2 $2^{\mathrm{V} 617 \mathrm{~F}}\left(\mathrm{Ba} / \mathrm{F} 3-\mathrm{JAK} 2^{\mathrm{V} 617 \mathrm{~F}}\right)$. Surprisingly, in all of these JAK2 ${ }^{\mathrm{V} 617 \mathrm{~F}}$-harboring cells, SB1518 led to an increase of pJAK2 (Y1007/8) levels (Figures 2a and b). To further investigate whether this phenomenon is a general effect of JAK2 inhibitors, HEL92.1.7 cells were treated for $3 \mathrm{~h}$ with JAK inhibitor-1, TG101348 and INCB018424. Similar to our findings with SB1518, all three JAK2 kinase inhibitors increased pJAK2 (Y1007/8) levels (Figure 2c). A time course was performed in HEL92.1.7 cells to further investigate the unexpected increase of pJAK2 (Y1007/8). Treatment of the cells with SB1518 led to a continuous increase of pJAK2 (Y1007/8) for up to $24 \mathrm{~h}$ (Figure 2d).

JAK2 has been described to possess additional autophosphorylation sites other than $Y 1007 / 8$. Therefore, the change in phosphorylation of $\mathrm{Y} 1007 / 8$ was compared with Y221, which is critically involved in the activation of JAK2. ${ }^{13}$ Interestingly, Y221 phosphorylation could be blocked by SB1518, even though Y1007/8 was increased (Figure 2e). Furthermore, immunoprecipitated JAK2 showed an increased JAK2 (Y1007/8) phosphorylation, but a decrease in total tyrosine phosphorylation following SB1518 treatment in JAK2 ${ }^{\mathrm{V} 617 \mathrm{~F}}$-bearing cell lines (Supplementary Figure S2).

Despite an increase in pJAK2 (Y1007/8) in cells bearing the V617F mutant, a clear evidence of JAK2 signaling blockade is shown by a dose-dependent reduction of pSTAT3 and pSTAT5 following SB1518 treatment of SET-2 and HEL92.1.7 cells
(Figures 2a/b). SB1518 led to a similar increase in pJAK2 (Y1007/8) and reduction in the phosphorylation of STAT5 in murine Ba/F3-JAK2 ${ }^{\mathrm{V} 617 \mathrm{~F}}$ cells (data not shown). Thus, SB1518 consistently shows the ability to attenuate signaling downstream of endogenously or ectopically expressed JAK2 ${ }^{\mathrm{V} 617 \mathrm{~F}}$ even though phosphorylation of $\mathrm{Y} 1007 / 8$ is increased.

Overall, these data show that SB1518 effectively permeates cells to modulate signaling pathways downstream of JAK2, whether agonist activated or mutationally activated.

\section{SB1518 induced apoptosis, cell cycle arrest and antiproliferative effects in JAK2 ${ }^{W T}$ - and JAK2 $2^{V 617 F}$-dependent cells}

As JAK2 signaling pathways are known to be key regulators of important functional responses, the effects of JAK2 inhibition on apoptosis and cell cycle were investigated. SET-2 and Karpas $1106 \mathrm{P}$ cells were treated with SB1518 for 16 or $24 \mathrm{~h}$ and analyzed for caspase-3/7 activation and cell cycle arrest, respectively. The ability of SB1518 to induce apoptosis is shown in Figure 3a where caspase-3/7 was dose dependently activated after $16 \mathrm{~h}$ of drug exposure. Figure $3 \mathrm{~b}$ showed that SB1518 arrested both JAK2 ${ }^{\mathrm{WT}}$ - and JAK2 ${ }^{\mathrm{V} 617 \mathrm{~F}}$-expressing cells at $\mathrm{G}_{0 / 1}$ phase and decreased the S-phase population after $24 \mathrm{~h}$ exposure in a concentration-dependent manner. In conclusion, SB1518 inhibition of JAK2 pathways in cells can lead to the induction of apoptosis and arrest of cell cycle at G1 phase.

Because the observed functional changes in cell cycle and apoptosis are expected to have an impact on cell proliferation, the effect on proliferation was studied in 25 cell lines derived from both liquid and solid tumors of human or murine origin (Figure 3c). Consistent with the kinase selectivity profile of SB1518, the sensitive cell lines are the ones known to express hyperactivated forms of JAK2 or FLT3 or known to be growth dependent on cytokines that signal primarily through JAK2.
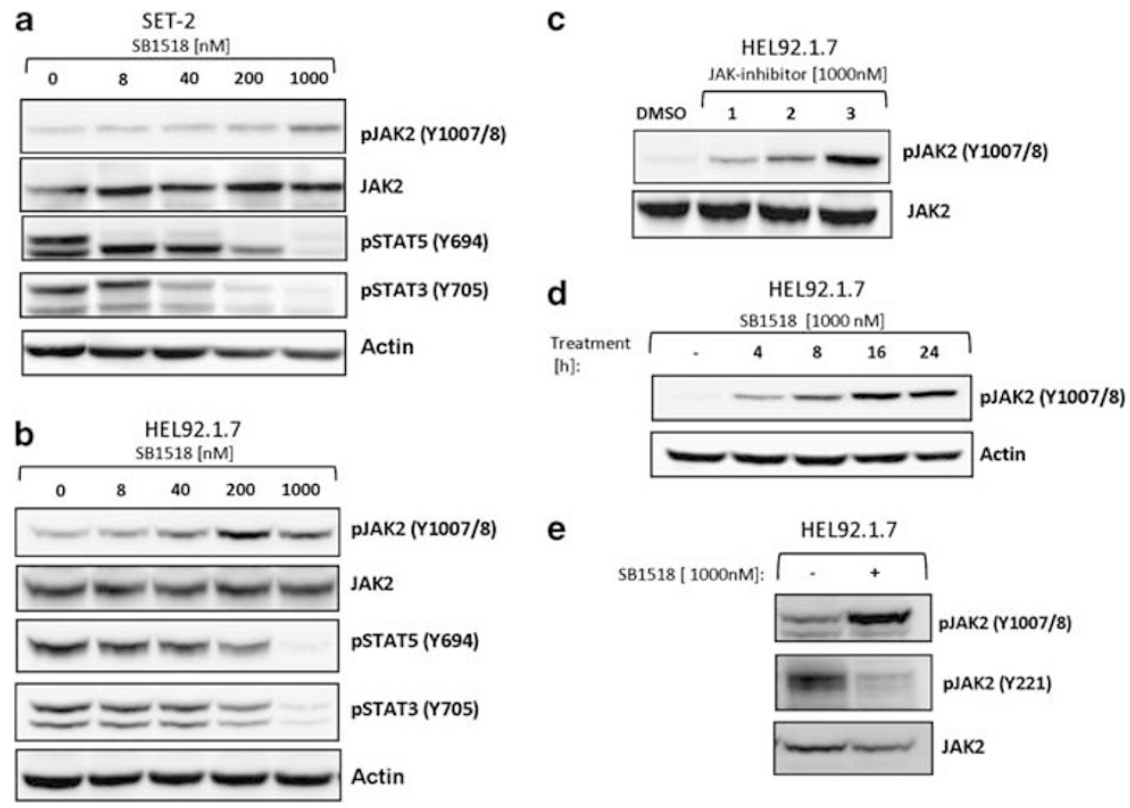

Figure 2 SB1518 effectively blocks signaling pathways in JAK2 ${ }^{\mathrm{V} 617 \mathrm{~F}}$-expressing cell lines. (a) SET-2 and (b) HEL92.1.7 cells were treated for $3 \mathrm{~h}$ with SB1518. After lysis, pJAK2 (Y1007/8) was detected by immunoblotting (IB) with anti-p(1007/8)JAK2 antibody, followed by re-probing of the same membrane with anti-JAK2, anti-pSTAT3(Y705), anti-pSTAT5(Y694) and anti-actin antibody. (c) HEL92.1.7 cells were treated for $3 \mathrm{~h}$ with three JAK2 inhibitors (1, TG101348; 2, INCB018424; 3, JAK inhibitor-1) as indicated and pJAK2 (Y1007/8) and total JAK2 were detected by immunoblotting. (d) HEL92.1.7 cells were treated with $1 \mu \mathrm{M}$ SB1518 for the indicated times and pJAK2 (Y1007/8) and JAK2 were detected by IB. (e) HEL92.1.7 cells were treated with $1 \mu \mathrm{M}$ SB1518 for $3 \mathrm{~h}$ and pJAK2 (Y1007/8), pJAK2 (Y221) and JAK2 were detected by IB. 

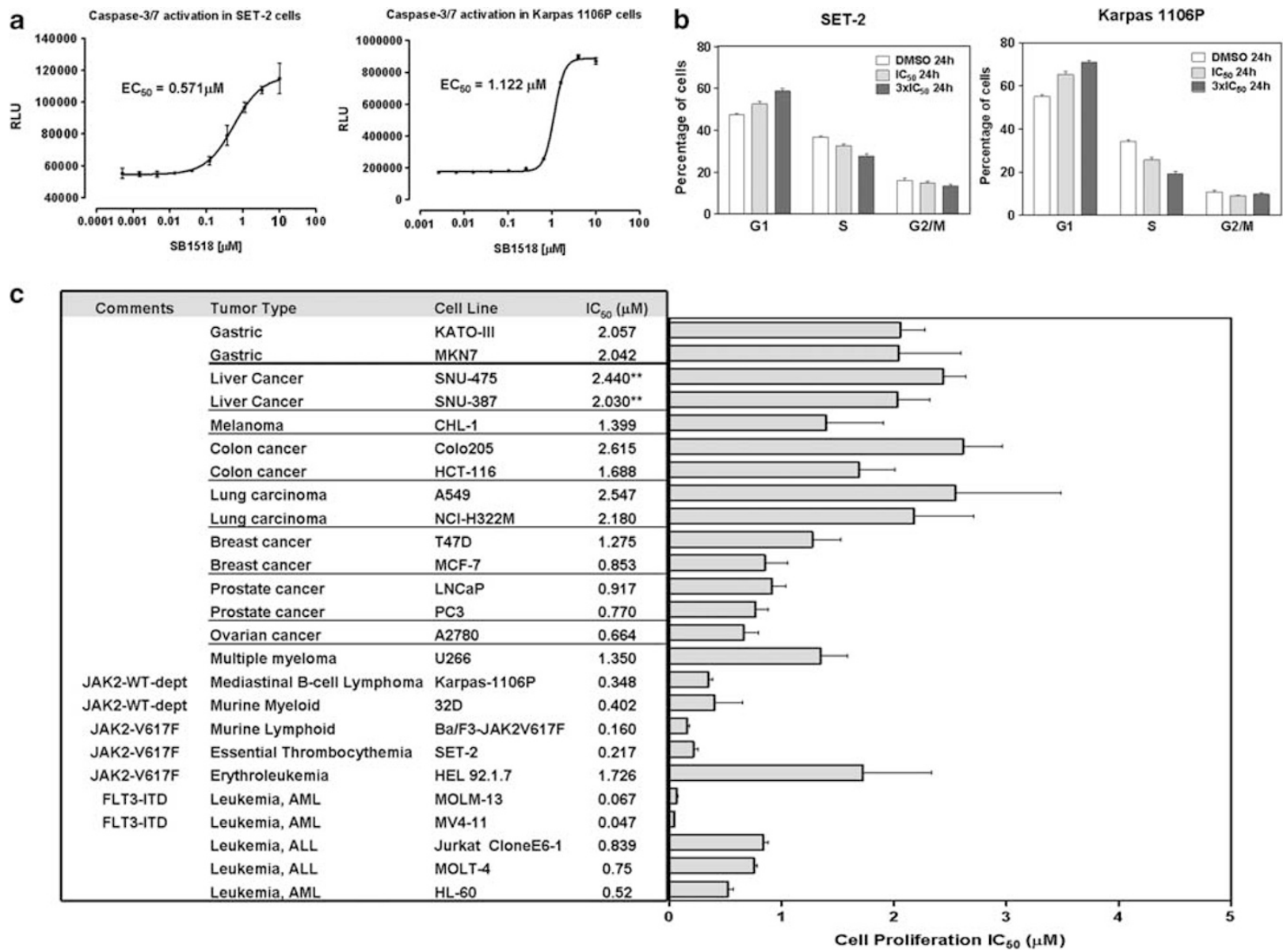

Figure 3 SB1518 induces apoptosis, cell cycle arrest and inhibition of proliferation in cancer cells. (a) SET-2 and Karpas 1106P cells were treated for $16 \mathrm{~h}$ with SB1518, and caspase-3/7 activity was determined. (b) SET-2 and Karpas $1106 \mathrm{P}$ cells were treated for $24 \mathrm{~h}$ with SB1518 at $1 \times$ and $3 \times \mathrm{IC}_{50}$ and the cell cycle analysis was performed using propidium-iodine staining followed by flow cytometric measurement. (c) To determine the cell proliferation $\mathrm{IC}_{50}$ values, cells were treated for $48 \mathrm{~h}$ with various concentrations of SB1518 and their viability tested with CellTiterGlo Assay (Promega). The mean IC $_{50}$ results were calculated from four independent experiments. The error bars on bar graph denote s.d.'s. **For SNU-387 and SNU-475, $n=2$ and the error bars denote data spread.

The cell lines with highest sensitivity in our panel were MV4-11 and MOLM-13 $\left(\mathrm{IC}_{50}=47\right.$ and $67 \mathrm{nM}$, respectively), both of which are cell lines derived from human acute myeloid leukemias driven by an FLT3 ITD mutation. Karpas 1106P and $\mathrm{Ba} / \mathrm{F} 3-\mathrm{JAK} 2^{\mathrm{V} 617 \mathrm{~F}}$, which are cell lines dependent on JAK2 signaling, show an $\mathrm{IC}_{50}$ of 348 and $160 \mathrm{nM}$, respectively.

\section{Therapeutic effects of SB1518 in a JAK2 $2^{\mathrm{V} 617 F}$-dependent xenograft model}

Several groups have reported an aggressive disease model in mice whereby Ba/F3-JAK2 ${ }^{\mathrm{V} 617 \mathrm{~F}}$ cells, murine cells of lymphoid origin, are injected intravenously into the animals to induce hallmark symptoms of myelofibrosis, such as progressive splenomegaly and hepatomegaly rapidly leading to end-stage hematopoietic crisis and death. ${ }^{14,15}$ To ensure target inhibition over $24 \mathrm{~h}$ in the Ba/F3-JAK2 ${ }^{\mathrm{V} 617 \mathrm{~F}}$ disease model, the PK profile was determined in this model (Supplementary Figure S1) and a maximum dose of $150 \mathrm{mg} / \mathrm{kg}$ p.o. q.d. was chosen for the subsequent efficacy studies. For evaluation of efficacy in the $\mathrm{Ba} / \mathrm{F} 3-\mathrm{JAK} 2^{\mathrm{V} 617 \mathrm{~F}}$ engraftment model, mice were treated with SB1518 at doses of 50 or $150 \mathrm{mg} / \mathrm{kg}$ p.o. q.d. for 13 days, with drug dosing starting 4 days after cell inoculation. At study termination, the vehicle control mice exhibited splenomegaly and hepatomegaly ( $\sim 7$ - and 1.3-fold, respectively, Figure $4 a)$, reminiscent of the symptoms found in patients with symptomatic myelofibrosis. SB1518 treatment at $150 \mathrm{mg} / \mathrm{kg}$ p.o. q.d. significantly ameliorated all these symptoms, with $60 \%( \pm 9 \%)$ normalization of spleen weight and $92 \%( \pm 5 \%)$ normalization of liver weight (Figure 4a) and was well tolerated without significant weight loss or any hematological toxicities, including thrombocytopenia and anemia (Figure 4b).

Pharmacodynamic and therapeutic effects of SB1518 in a JAK2 ${ }^{V 617 F}$-dependent SET-2 xenograft model

To further investigate the pharmacodynamic and therapeutic effects of SB1518 on JAK2 ${ }^{\mathrm{V} 617 \mathrm{~F}}$-driven malignancy in vivo, a xenograft model using subcutaneous injection of SET-2 cells (derived from a JAK2 $2^{\mathrm{V} 617 \mathrm{~F}}$ patient with essential thrombocythemia who had progressed to megakaryoblastic leukemia) was used. In the first experiment, mice with an average tumor size of $300 \mathrm{~mm}^{3}$ were given single oral doses of SB1518 (75 and $150 \mathrm{mg} / \mathrm{kg}$ ). Tumor biopsies were collected and lysed $3 \mathrm{~h}$ after dosing to study the effects of treatment on JAK2 signaling. Consistent with the in vitro cellular studies, SB1518 increased pJAK2 $^{\mathrm{V} 617 \mathrm{~F}}$ (Y1007/8) in the tumor tissue, but simultaneously inhibited phosphorylation of STAT5 in a dose-dependent 

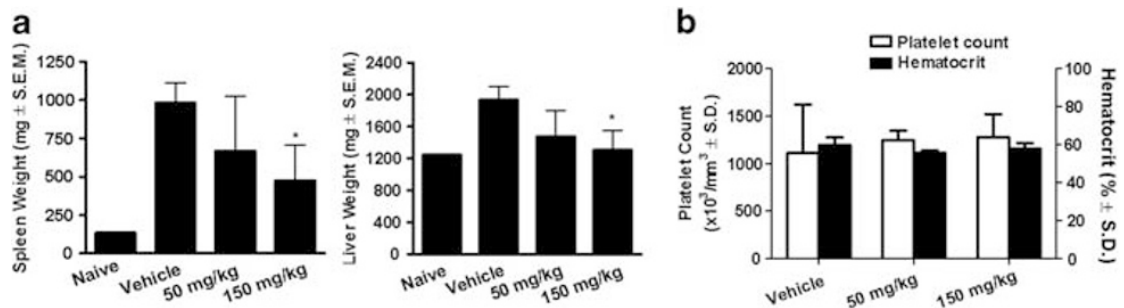

Figure 4 SB1518 reduces splenomegaly and hepatomegaly in Ba/F3-JAK2 ${ }^{\mathrm{V} 617 \mathrm{~F}}$ xenograft model. Ba/F3-JAK2 ${ }^{\mathrm{V} 617 \mathrm{~F}}$-bearing nude mice were dosed p.o. q.d. with 50 and $150 \mathrm{mg} / \mathrm{kg} \mathrm{SB} 1518$ for 12 consecutive days. On day 12, mice were killed and (a) spleen and liver weights determined ( $n=6$; ${ }^{*} P<0.05$, analysis of variance, Dunnett's post test) (b) and platelet counts and hematocrit measured.
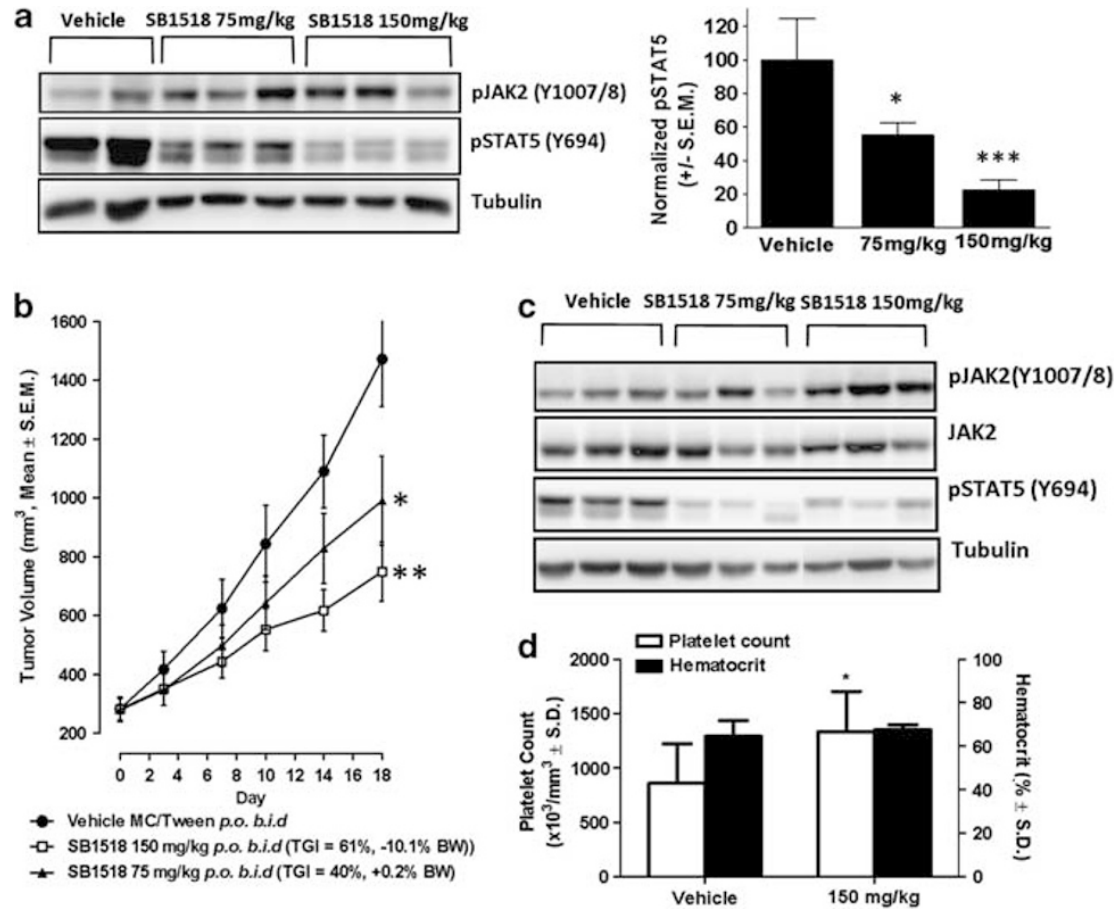

Figure 5 SB1518 inhibits JAK2 signaling and tumor growth in a SET-2 xenograft model of myeloproliferative neoplasm. (a) SET-2 tumor-bearing mice were dosed p.o. once with 75 and $150 \mathrm{mg} / \mathrm{kg}$ SB1518. Three hours after dosing, mice were killed, tumors lysed; pJAK2 (Y1007/8), pSTAT5(Y694) and tubulin were analyzed by immunoblotting (IB; left panel); and densitometric analysis was performed (right panel). ${ }^{*} P<0.05$ ${ }^{* * *} P<0.001$; analysis of variance, Dunnett's post test. (b) SCID Beige mice bearing s.c. SET-2 tumors $(n=12)$ were treated for 18 consecutive days with 75 and $150 \mathrm{mg} / \mathrm{kg}$ SB1518 p.o. b.i.d. Tumor volumes were measured twice a week. ${ }^{*} P<0.05,{ }^{* *} P<0.01$, analysis of variance, Dunnett's post test, BW, body weight. (c) $3 \mathrm{~h}$ after dosing on day 18, mice were killed and tumor lysates were analyzed for JAK2 signaling by Western blot. Representative data for three mice/group are shown (d) At day 18, the platelet counts of the vehicle group and the SB1518-treated group $\left(150 \mathrm{mg} / \mathrm{kg}\right.$ p.o. b.i.d.) were measured $\left({ }^{*} P<0.05, \mathrm{t}\right.$-test).

manner, indicating effective blockade of JAK2 signaling (Figure 5a).

Having demonstrated that SB1518 blocks JAK2 signaling in tumor tissues in the SET-2 model, the drug was then evaluated for its antitumor efficacy. SET-2 tumor-bearing mice (average starting tumor size of $130 \mathrm{~mm}^{3}$ ) were treated for 18 consecutive days. SB1518 treatment induced dose-dependent inhibition of tumor growth $(40 \%$ for $75 \mathrm{mg} / \mathrm{kg}$ and $61 \%$ for $150 \mathrm{mg} / \mathrm{kg}$, Figure $5 \mathrm{~b}$ ). In addition, analysis of JAK2 signaling pathway in the tumor lysates $3 \mathrm{~h}$ after the last treatment on day 18 showed a full inhibition of STAT5 phosphorylation (Figure 5c). These therapeutic effects of SB1518 treatment were achieved without thrombocytopenia, anemia (Figure 5d) or leukopenia (data not shown).

In conclusion, the inhibition of JAK2 kinase by SB1518 in a SET-2 xenograft model resulted in an efficient blockade of
STAT5 phosphorylation in tumor tissues and significant inhibition of tumor growth.

Effects of SB1518 on ex vivo expanded primary EPCS Having demonstrated inhibition of disease progression and tumor growth in xenograft models generated with transformed cell lines, it was of interest to investigate whether SB1518 treatment could also inhibit viability of primary cells from patients with JAK2-driven disease. EPCs present in PBMCs of $12 \mathrm{PV}$ patients $\left(11 \mathrm{JAK} 2^{\mathrm{V} 617 \mathrm{~F}}, 1 \mathrm{JAK} 2^{\mathrm{WT}}\right.$, see Figure $\left.6 \mathrm{~b}\right)$ or 8 healthy donors (JAK2 ${ }^{\mathrm{WT}}$ ) were expanded over 12 days. Flow cytometry confirmed that $>98 \%$ of the cell population at day 12 of the EP expansion protocol belonged to the basophilic erythroblast lineage, expressing both the CD71 and CD235a markers (Supplementary Figure S3A). To identify a possible role 

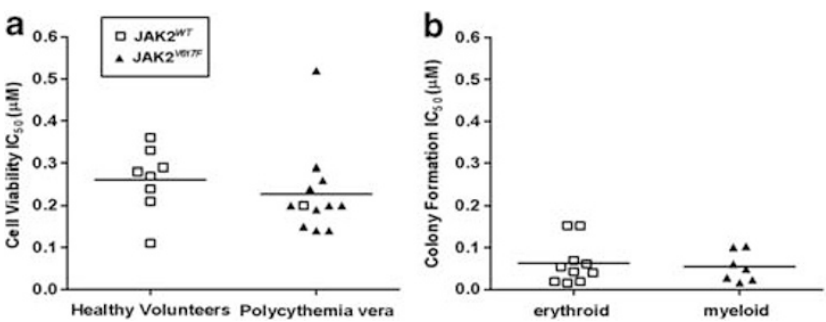

Figure 6 The effect of SB1518 on ex vivo expanded erythroid progenitor cells (EPCs). (a) On day 12 of the ex vivo expansion protocol of EPCs from peripheral blood mononuclear cells (PBMCs) from polycythemia vera patients and normal volunteers, cells were treated with increased concentrations of SB1518 for $48 \mathrm{~h}$ and the $\mathrm{IC}_{50}$ on cell viability was evaluated using the CellTiter-Glo assay. (b) On day 14 after seeding PBMCs in the Metholcult media, erythroid (BFU-E and CFU-E) and myeloid (CFU-M and CFU-GM) were enumerated. The range of erythroid and myeloid colonies per plate was 17-122 and $12-32$, respectively.

for JAK2 signaling in the viability of EP, the cells were treated with SB1518 for $48 \mathrm{~h}$. Our data showed that the inhibitory effect of SB1518 on the viability of the progenitors did not depend on the JAK2 mutation status. There was no significant difference in the average $\mathrm{IC}_{50}$ values for samples from PV patients $(0.26 \mu \mathrm{M})$ compared with the samples from healthy volunteers $(0.23 \mu \mathrm{M}$; Figure 6a). Phosphorylation of STAT5 was also dose-dependently inhibited to a similar extent in EP from both PV patients and healthy volunteers (Supplementary Figure S3B). Furthermore, we showed that EP exposed to SB1518 for $16 \mathrm{~h}$ underwent apoptosis as indicated by the dose-dependent activation of caspase-3/7 (Supplementary Figure S3C). JAK2 ${ }^{\mathrm{V} 617 \mathrm{~F}}$-positive EPCs exhibit the potential to form colonies in vitro in the absence of EPO. Therefore, we cultivated PBMCs derived from peripheral blood of PV patients in the absence of cytokines and assessed the effect of SB1518 treatment. The mean $\mathrm{IC}_{50}$ on endogenous colony growth derived from erythroid and myeloid progenitors were 63 and $53 \mathrm{nM}$, respectively (Figure 6b). These results strengthen the notion that the JAK2 pathway has a crucial role in progenitor cell viability and endogenous colony formation in PV patients harboring the JAK2 ${ }^{\mathrm{V} 61 \text { FF }}$ mutation.

\section{Discussion}

Myelofibrosis is an indication with unmet medical need and the discovery of a widespread somatic mutation (JAK2 $\left.{ }^{\mathrm{V} 617 \mathrm{~F}}\right)$ in this and other Ph-negative myeloproliferative neoplasms engendered hopes of a targeted therapy that will emulate the success of imatinib in chronic myelogenous leukemia treatment. Here we present for the first time the preclinical evaluation of a novel chemical entity, SB1518, a small molecule designed as an adenosine triphosphate-competitive JAK2 inhibitor. Although the pyrimidine-based core of SB1518 is found in other kinase inhibitors, its macrocyclic nature is innovative, ${ }^{16}$ leading to a unique and clinically interesting kinase spectrum. In vitro kinase data show selectivity for JAK2 over other members of the JAK family with highest selectivity against JAK1 and least against TYK2. Compared with other JAK2 clinical candidates, including INCB018424, TG101348, AZD1480 and CYT387, ${ }^{17}$ SB1518 showed the highest selectivity for JAK2 compared with JAK1 (56-fold). The favorable preclinical data described here as well as the emerging clinical data with SB1518 and others such as TG101348, indicate that JAK1 activity is not mandatory for therapeutic benefit in MPDs. ${ }^{18-20}$ It remains to be determined how the presence or absence of JAK1 activity contributes to a different clinical efficacy and safety profile. An additional feature of SB1518 is the potent activity against FLT3 and its drug-resistant D835Y mutant, but without activity against class III RTKs in the same concentration range. ${ }^{3}$ Furthermore, the highest antiproliferative potency of SB1518 is against cells with either activated JAK2 or FLT3 signaling indicates that JAK2 and FLT3 are the key targets for the therapeutic efficacy of SB1518 (Figure 3c). The interesting JAK2/FLT3 inhibitory profile has the potential to broaden therapeutic indications beyond myeloid and lymphoid malignancies and data supporting this will be published in a separate manuscript.

SB1518 shows effective inhibition of the JAK/STAT signaling pathways in JAK2 ${ }^{\mathrm{WT}}$ - or JAK2 ${ }^{\mathrm{V} 617 \mathrm{~F}}$-expressing cell lines. Importantly, cytokine-induced JAK/STAT signaling was shown to be inhibited by SB1518 at the same dose level compared with basal JAK/STAT signaling arising from the constitutively activated kinase induced via a JAK2 mutation. Surprisingly, in contrast to JAK2 ${ }^{\mathrm{WT}}$-expressing cells, JAK2 ${ }^{\mathrm{V} 617 \mathrm{~F}}$-harboring cells showed increased levels of phosphorylation of JAK2 at the Y1007/8 residue on exposure to SB1518. However, effective inhibition of downstream signaling events, namely STAT3/5 phosphorylation, was observed. Concomitantly, the phosphorylation of JAK2 at tyrosine 221, which is suggested to be necessary for JAK2 to attain full activity, ${ }^{13}$ was fully blocked by SB1518 treatment (Figure 2e). We also observed an increase in Y1007/8 phosphorylation with three other structurally unrelated JAK2 inhibitors despite inhibition of downstream STAT5 signaling (Figure 2c). Furthermore, inhibition of downstream STAT signaling and a concurrent increase of pJAK2 (Y1007/8) have been reported using the JAK2 inhibitor Gö6976 in the same cellular context (HEL92.1.7). ${ }^{18}$ The authors proposed that this JAK2 inhibitor preferentially binds to and inhibits a 'preactivated' form of JAK2, already phosphorylated at Y1007/8, leading to an increased level of this species of pJAK2 protein. SB1518, being a Type I inhibitor, ${ }^{19}$ only binds to the active state ('DFG-in' conformation) of tyrosine kinases. Tyrosine phosphorylation such as pJAK2-Y1007/8 in the activation loop may stabilize the active state of the kinase and allow SB1518 to bind and therefore block any further substrate phosphorylation. ${ }^{20}$ JAK2 ${ }^{\mathrm{V} 617 \mathrm{~F}}$ is described to escape negative regulation by SOCS3 and this leads to the increase of phosphorylated, but inactive JAK2 $2^{\mathrm{V} 617 \mathrm{~F}}$ in JAK2 mutant cell lines after JAK2 inhibitor treatment. ${ }^{21}$ The inability of the cells to downregulate JAK2 ${ }^{\mathrm{V} 617 \mathrm{~F}}$ is also supported by our observations that pJAK2 ${ }^{\mathrm{V} 617 \mathrm{~F}}$ (Y1007/8) accumulates in the cells for up to $24 \mathrm{~h}$ (Figures $2 \mathrm{~d} / \mathrm{e}$ ). Consistent with our observation, Tyner et al. ${ }^{22}$ observed an increased level of pJAK2 (Y1007/8) when treating Ba/F3-JAK2 ${ }^{\mathrm{V} 617 \mathrm{~F}}$ cells with yet another JAK2 inhibitor and also postulated that this molecule may bind to an open phosphorylated form of JAK2. Hence, the increase of the phosphorylation of Y1007/8 of JAK2 appears to be a class effect induced by adenosine triphosphate-competitive JAK2 inhibitors.

Inhibition of the JAK-STAT pathway by SB1518 resulted in apoptotic induction and cell cycle arrest in both cytokineactivated JAK2 ${ }^{\mathrm{WT}}$ - or JAK2 ${ }^{\mathrm{V} 617 \mathrm{~F}}$-expressing cell lines. Our data show that, independent of JAK2 mutation status, SB1518 can lead to cell cycle arrest in JAK2-dependent cell lines. Cell proliferation assays showed that SB1518 exhibits greater antiproliferative activity in cell lines with hyperactivated forms of JAK2 or activating FLT3 mutations (Figure 3c). The potent inhibition of proliferation of Ba/F3-JAK2 ${ }^{\mathrm{V} 617 \mathrm{~F}}$ cells, which are dependent on JAK2 signaling, is also reported for other JAK2 kinase inhibitors. ${ }^{14,23}$ In contrast, HEL92.1.7 cells that are homozygous for JAK2 ${ }^{\mathrm{V} 617 \mathrm{~F}}$ and have been used extensively for 
signaling studies in the JAK2 field show potent inhibition of STAT signaling after treatment with JAK2 inhibitors, but are relatively less sensitive to the antiproliferative effects of SB1518 $\left(\mathrm{IC}_{50}=1.7 \mu \mathrm{M}\right)$. The weaker antiproliferative activity in HEL92.1.7 compared with other JAK2 ${ }^{\mathrm{V} 617 \mathrm{~F}}$ mutant cell lines is also reported for other JAK2 inhibitors such as CYT387 $\left(I C_{50}=1.5 \mu \mathrm{M}\right)^{22}$ and XL019 $\left(I_{50}=6.8 \mu \mathrm{M}\right){ }^{24}$ This suggests that the proliferative signals for these cells may originate from pathways other than JAK2/STAT.

Kinase activity of JAK2 in cells is negatively regulated by factors including a group of proteins known as SOCS. In primary lymphoma specimens as well as lymphoma cell lines, mutations of the SOCS-1 gene have been found to occur frequently and result in the increased stabilization and concomitant activation of wild-type JAK2. ${ }^{12,25}$ In this regard, it is interesting that SB1518 shows antiproliferative activity on Karpas 1106P, a lymphoma cell line reported to possess such a defect in the SOCS1-JAK2 axis. ${ }^{12}$ Further evidence for a pivotal role of JAK2 in lymphoma progression is supported by the discovery that a high level of a microRNA species targeting JAK2 (miR-135a) correlates with a favorable prognosis in classical Hodgkin lymphoma patients. ${ }^{26}$ Amplification of 9p.24 locus containing the JAK2 gene were detected in various lymphoma subtypes, including primary mediastinal B-cell lymphomas, Hodgkin's lymphomas and angioimmunoblastic T-cell lymphomas. ${ }^{27,28}$ Our cellular data, combined with these published observations, provide a rationale for clinical trials with SB1518 in lymphoma and may account for some of the promising efficacy, including partial responses, observed in ongoing phase 1 trials. ${ }^{5,29}$

Our animal model data provide further rationale for the clinical trials with SB1518 in patients with JAK2-driven disease. In a murine model with symptoms of myeloproliferative disease established after intravenous injection of $\mathrm{Ba} / \mathrm{F} 3$ cells with exogenously expressed JAK2 ${ }^{\mathrm{V} 617 \mathrm{~F}}$, we showed significant amelioration of the disease symptoms of hepatomegaly and splenomegaly after oral dosing of SB1518. Ba/F3 cells are of lymphoid origin and the organomegaly seen in these models are symptoms also found in certain lymphomas. ${ }^{30}$ Data from these models therefore suggest potential in lymphoid as well as myeloid disease driven by JAK2 signaling. SET-2 cells are megakaryoblastic acute myeloid leukemia cells derived from a patient originally diagnosed with ET and have endogenous JAK2 ${ }^{\mathrm{V} 617 \mathrm{~F}}$. They are a cytokine-independent cell line showing a low expression of the negative regulator SOCS-2. ${ }^{31}$ These cells provided us an alternative model for a JAK2-driven disease and we could show that SB1518 potently blocked JAK/STAT signaling in tumor tissue after both acute and chronic dosing (Figures 5a and c). SB1518 also effectively blocked tumor growth in a dose-dependent manner. In all the animal models tested, SB1518 was well tolerated with minimal body weight loss and with no hematological toxicity. In particular, thrombocytopenia and anemia were not observed at therapeutic doses.

Finally, support for clinical trials with SB1518 in patients with JAK2-driven disease come from our studies with expanded EPCs derived from PV patients and healthy subjects. Our studies show that SB1518 treatment potently blocked endogenous erythroid as well as myeloid colony formation. Furthermore, cell viability in the presence of erythropoietin showed similar potency on the viability of EP from normal volunteers as well as from PV patients with or without a JAK2 mutation. This suggests that the constitutively activated JAK2 mutant and the EPO-activated wild-type JAK2 exert a quantitatively equivalent 'driver' role on proliferative pathways in EP. These data are consistent with current observations in trials with various JAK2 inhibitors in patients with myeloproliferative disease, namely that clinical benefit is attainable independent of the patient's JAK2 mutation status. In view of this, we postulate that SB1518, being equipotent against both WT and mutant JAK2 kinases, will exert comparable effects in disease driven by JAK2 activation, whether constitutive or ligand stimulated.

In summary, SB1518, a novel chemical entity selective for JAK2 over JAK1 and JAK3 with additional activity against FLT3, is efficacious against cell lines dependent on constitutively active or ligand-activated JAK2 and FLT3 signaling and effectively blocks STAT signaling in these cells. SB1518 is orally active, efficacious and well tolerated in models of JAK2- and FLT3-driven disease. ${ }^{32}$ SB1518's favorable pharmaceutical and pharmacological properties provide a rationale for clinical development in multiple myeloid and lymphoid disease indications. Promising data for SB1518 have recently been reported after treatment of patients with myelofibrosis and lymphoma, showing successful translation of preclinical findings into the clinic. $5,6,29,33$

\section{Conflict of interest}

With the exception of $\mathrm{KHO}$, all authors are current or past full-time employees of $\mathrm{S} * \mathrm{BIO}$.

\section{Acknowledgements}

We are grateful to Dr Martin Sattler (Dana-Farber Cancer Institute, Boston) for the Ba/F3-JAK2 ${ }^{\mathrm{V} 617 \mathrm{~F}}$ cells and to $\mathrm{Ai}$ Leng Liang, Venkatesh Reddy and Evelyn Goh for technical assistance.

\section{References}

1 Rawlings JS, Rosler KM, Harrison DA. The JAK/STAT signaling pathway. J Cell Sci 2004; 117(Pt 8): 1281-1283.

2 Bennett M, Stroncek DF. Recent advances in the bcr-abl negative chronic myeloproliferative diseases. J Transl Med 2006; 4: 41.

3 Tickenbrock L, Muller-Tidow C, Berdel WE, Serve H. Emerging Flt3 kinase inhibitors in the treatment of leukaemia. Expert Opin Emerg Drugs 2006; 11: 153-165.

4 Driggers EM, Hale SP, Lee J, Terrett NK. The exploration of macrocycles for drug discovery - an underexploited structural class. Nat Rev Drug Discov 2008; 7: 608-624.

5 Younes A, Fanale MA, McLaughlin P, Copeland A, Zhu J, Faria SdC. Phase I study of a novel oral JAK-2 inhibitor sb1518 in patients with relapsed lymphoma: evidence of clinical and biologic activity in multiple lymphoma subtypes. Blood 2010; 116: Abstract 2830.

6 Verstovsek S, Deeg HJ, Odenike O, Zhu J, Kantarjian HM, Estrov Z et al. Phase 1/2 study of SB1518, a novel JAK2/FLT3 inhibitor, in the treatment of primary myelofibrosis. Blood 2010; 116: Abstract 3082.

7 Walz C, Crowley BJ, Hudon HE, Gramlich JL, Neuberg DS, Podar $\mathrm{K}$ et al. Activated Jak2 with the V617F point mutation promotes G1/S phase transition. J Biol Chem 2006; 281: 18177-18183.

8 Manshouri T, Quintas-Cardama A, Nussenzveig RH, Gaikwad A, Estrov Z, Prchal J et al. The JAK kinase inhibitor CP-690,550 suppresses the growth of human polycythemia vera cells carrying the JAK2V617F mutation. Cancer Sci 2008; 99: 1265-1273.

9 Borrell-Pages M, Rojo F, Albanell J, Baselga J, Arribas J. TACE is required for the activation of the EGFR by TGF-alpha in tumors. EMBO J 2003; 22: 1114-1124.

10 Novotny-Diermayr V, Sangthongpitag K, Hu CY, Wu X, Sausgruber N, Yeo P et al. SB939, a novel potent and orally active histone deacetylase inhibitor with high tumor exposure and efficacy in mouse models of colorectal cancer. Mol Cancer Ther 2010; 9: 642-652. 
11 van Boxel-Dezaire AH, Stark GR. Cell type-specific signaling in response to interferon-gamma. Curr Top Microbiol Immunol 2007; 316: 119-154.

12 Melzner I, Weniger MA, Bucur AJ, Bruderlein S, Dorsch K, Hasel C et al. Biallelic deletion within 16p13.13 including SOCS-1 in Karpas1106P mediastinal B-cell lymphoma line is associated with delayed degradation of JAK2 protein. Int J Cancer 2006; 118: $1941-1944$

13 Argetsinger LS, Kouadio JL, Steen H, Stensballe A, Jensen ON, Carter-Su C. Autophosphorylation of JAK2 on tyrosines 221 and 570 regulates its activity. Mol Cell Biol 2004; 24: 4955-4967.

14 Pardanani A, Hood J, Lasho T, Levine RL, Martin MB, Noronha G et al. TG101209, a small molecule JAK2-selective kinase inhibitor potently inhibits myeloproliferative disorder-associated JAK2V617F and MPLW515L/K mutations. Leukemia 2007; 21: 1658-1668.

15 Quintas-Cardama A, Vaddi K, Liu P, Manshouri T, Li J, Scherle PA et al. Preclinical characterization of the selective JAK1/2 inhibitor INCB018424: therapeutic implications for the treatment of myeloproliferative neoplasms. Blood 2010; 115: 3109-3117.

16 William A, Lee A, Blanchard S, Poulsen A, Teo E, Nagaraj H et al. Discovery of the macrocycle SB1518, a potent JAK2/FLT3 inhibitor for the treatment of myelofibrosis and lymphoma. J Med Chem 2011; dx.doi.org/10.1021/jm200326p.

17 Tefferi A. How I treat myelofibrosis. Blood 2011; 117: 3494-3504.

18 Grandage VL, Everington T, Linch DC, Khwaja A. Gö6976 is a potent inhibitor of the JAK 2 and FLT3 tyrosine kinases with significant activity in primary acute myeloid leukaemia cells. Br J Haematol 2006; 135: 303-316.

19 Zuccotto F, Ardini E, Casale E, Angiolini M. Through the 'gatekeeper door': exploiting the active kinase conformation. J Med Chem 2010; 53: 2681-2694.

20 Lucet IS, Fantino E, Styles M, Bamert R, Patel O, Broughton SE et al. The structural basis of Janus kinase 2 inhibition by a potent and specific pan-Janus kinase inhibitor. Blood 2006; 107: 176-183.

21 Hookham MB, Elliott I Suessmuth Y, Staerk I, Ward AC Vainchenker $\mathrm{W}$ et al. The myeloproliferative disorder-associated JAK2 V617F mutant escapes negative regulation by suppressor of cytokine signaling 3. Blood 2007; 109: 4924-4929.

22 Tyner JW, Bumm TG, Deininger J, Wood L, Aichberger KJ Loriaux MM et al. CYT387, a novel JAK2 inhibitor, induces hematologic responses and normalizes inflammatory cytokines in murine myeloproliferative neoplasms. Blood 2010; 115: 5232-5240.

23 Wernig G, Kharas MG, Okabe R, Moore SA, Leeman DS, Cullen DE et al. Efficacy of TG101348, a selective JAK2 inhibitor, in treatment of a murine model of JAK2V617F-induced polycythemia vera. Cancer Cell 2008; 13: 311-320.
24 Verstovsek S, Pardanani AD, Shah NP, Sokol L, Wadleigh M, Gilliland DG et al. A phase I study of XL019, a selective JAK2 inhibitor, in patients with primary myelofibrosis and post-polycythemia vera/essential thrombocythemia myelofibrosis. Blood 2007; 110: Abstract 553.

25 Weniger MA, Melzner I, Menz CK, Wegener S, Bucur AJ, Dorsch K et al. Mutations of the tumor suppressor gene SOCS-1 in classical Hodgkin lymphoma are frequent and associated with nuclear phospho-STAT5 accumulation. Oncogene 2006; 25: 2679-2684.

26 Navarro A, Diaz T, Martinez A, Gaya A, Pons A, Gel B et al. Regulation of JAK2 by miR-135a: prognostic impact in classical Hodgkin lymphoma. Blood 2009; 114: 2945-2951.

27 Joos S, Granzow M, Holtgreve-Grez H, Siebert R, Harder L, Martin-Subero Jl et al. Hodgkin's lymphoma cell lines are characterized by frequent aberrations on chromosomes $2 \mathrm{p}$ and 9p including REL and JAK2. Int J Cancer 2003; 103: 489-495.

28 Meier C, Hoeller S, Bourgau C, Hirschmann P, Schwaller J, Went P et al. Recurrent numerical aberrations of JAK2 and deregulation of the JAK2-STAT cascade in lymphomas. Mod Pathol 2009; 22: 476-487.

29 Younes A, Fanale M, McLaughlin P, Copeland AR, Faria SdC, Wood I et al. Phase-I study of the novel oral JAK-2 inhibitor SB1518 in patients with relapsed lymphoma: evidence of clinical and biologic activity. Blood 2009; 114: Abstract 588.

30 Cheson BD. Staging and evaluation of the patient with lymphoma. Hematol Oncol Clin North Am 2008; 22: 825-837, vii-viii.

31 Quentmeier H, Geffers R, Jost E, Macleod RA, Nagel S, Rohrs S et al. SOCS2: inhibitor of JAK2V617F-mediated signal transduction. Leukemia 2008; 22: 2169-2175.

32 Hart S, Goh KC, Tan Y, Chithra A, Wood J. Pharmacodynamic (PD) biomarker assay validation for SB1518, a novel oral JAK2 inhibitor in phase I clinical trials for advanced leukemias, myeloproliferative diseases and lymphoma. Blood 2009; 114: Abstract 1888.

33 Verstovsek S, Odenike O, Scott B, Estrov Z, Cortes J, Thomas D et al. Phase I dose escalation trial of SB1518, a novel JAK2-FLT3 inhibitor, in acute and chronic myeloid diseases, including primary or post-ET/post-PV myelofibrosis. Blood 2009; 114: abstract 3905 .

(c) This work is licensed under the Creative Commons Attribution-NonCommercial-No Derivative Works 3.0 Unported License. To view a copy of this license, visit http:// creativecommons.org/licenses/by-nc-nd/3.0/

Supplementary Information accompanies the paper on the Leukemia website (http://www.nature.com/leu) 